\title{
Towards Fractal Gravity
}

\author{
Karl Svozil ${ }^{1}$ \\ Published online: 22 May 2019 \\ (C) The Author(s) 2019
}

\begin{abstract}
In an extension of speculations that physical space-time is a fractal which might itself be embedded in a high-dimensional continuum, it is hypothesized to "compensate" for local variations of the fractal dimension by instead varying the metric in such as way that the intrinsic (as seen from an embedded observer) dimensionality remains an integer. Thereby, an extrinsic fractal continuum is intrinsically perceived as a classical continuum. Conversely, it is suggested that any variation of the metric from its Euclidean (or Minkowskian) form can be "shifted" to nontrivial fractal topology. Thereby "holes" or "gaps" in spacetime could give rise to (increased) curvature.
\end{abstract}

Keywords Entanglement · Quantum state · Quantum indeterminism · Quantum randomness

Embedded observers and agents (Boskovich 1966; Toffoli 1978; Svozil 1994; Rössler 1998) are operationally bound by self-reflexive, intrinsic methods and means available from within the very system they exist. Such observers have no access to extrinsic, Platonistic entities which are beyond their operational physical capacities. [They may, nonetheless, have inspirational "afflatus" or ideas about some external truth; but would not be able to prove this in any effable way (Jonas 2016) beyond zero-knowledge proof methods.] Indeed the situation embedded observers have to cope with appear much more severe as in the allegory of the cave mentioned by Plato (2000, Book 7, 514a-517e, pp. 220-223), in that the latter assumes the existence of a supposedly ontologic level: an observer can be "dragged right out into the sunlight." The assumption of such ontologic level could, from an idealistic stance (Stace 1934), be considered problematic, as any observer appears to be permanently captivated in a Cartesian prison (Descartes 1996, Second Meditation, 26-29, pp. 17-20) [see also Putnam's "brain in the vat" metaphor (Putnam 1981, Chapter 1), among others], and "in the strict sense only a thing that thinks." As idealistic philosophy has it (Segal and Goldschmidt 2017), "the world is mental through-and-through." Poincaré has pointed out in the introduction to La valeur the la science (Poincaré 1905, 1913),

Karl Svozil

svozil@tuwien.ac.at

http://tph.tuwien.ac.at/ svozil

1 Institute for Theoretical Physics, Vienna University of Technology, Wiedner Hauptstrasse 8-10/136, 1040 Vienna, Austria 
"Does the harmony the human intelligence thinks it discovers in nature exist outside of this intelligence? No, beyond doubt a reality completely independent of the mind which conceives it, sees or feels it, is an impossibility. A world as exterior as that, even if it existed, would for us be forever inaccessible."

Therefore, when it comes to the formalization of physical theories, any such framework ought to include and use, as much as can be possibly afforded, intrinsic, that is, operationally feasible, elements of physical description (Bridgman 1934). Gaussian geometry, for example, characterizes a surface with totally intrinsic methods (Nottale 1993, Section 3.2, pp. 46, 47). It appears prudent to include epistemic considerations rather than uncritically assume that one deals with ontic elements of perception. Poincaré's and even more and explicitly so Einstein's conceptions and constructions of space and time follow this pursuit in that they operationalize physical time by conventionalizing, in particular, time synchronizations.

Nevertheless, quasi-extrinsic perspectives may shed new light on old physical subjects and concepts. Thereby, such extrinsic formalizations and situations, suggesting and utilizing means and methods available from a hypothetical outside, external viewpoint, may appear very different, even exotic and counterintuitive, from the point of view of embedded, intrinsic observers. In particular, based on Hausdorff measures and fractal dimension theory (Hurewicz and Wallman 1948; Rogers 1970; Kenneth 2014; Mattila 1995; Montiel et al. 1996; Adda 2007; Edgar 2008; Porchon 2012) of fractals (Mandelbrot 1982), it has been suggested that, while (i) extrinsically and ontologically, space-time might be a fractal set with possibly non-integer dimension (Ord 1983; Nottale and Schneider 1984), (ii) intrinsically and epistemically, that is, from an operational point of view, it might appear as if observers embedded in such fractals would experience not much phenomenological differences as compared to "inhabiting" standard continua such as $\mathbb{R}^{n}$ (Zeilinger and Svozil 1985; Svozil and Zeilinger 1986, 1988; Svozil 1986, 1987). In other words, the fractal space-time concept can be put to some extreme by speculating that, for all practical purposes, intrinsically embedded observers cannot differentiate between, say, three-dimensional continua $\mathbb{R}^{3}$ and some continuous fractal which is a (possibly stochastic) generalization of the Cantor set of fractal dimension three (Svozil 1986), and which is embedded in a larger-dimensional continuum, say, $\mathbb{R}^{d}$, with $d>3$.

I suggest here to take a further speculative step by shifting the nontrivial topological structure of such fractals to the metric of the (embedding) space. Because even for noninteger dimensions, intrinsic observers might, for all practical purposes, not be able to differentiate between two operationally indistinguishable premises: they may either exist in a space with standard (Euclidean, Minkowski) metric whose support is a fractal continuum; or they may inhabit a space-time whose support is a classical, integer dimensional continuum (say, $\mathbb{R}^{3}$ ), but the Riemannian metric of the space is somehow non-standard and, in particular, non-Euclidean or non-Minkowskian.

For the sake of an intuitive, informal example of why "cutting out holes" in a given set and "gluing together" the remaining pieces might affect the geometric properties of the object, consider a situation depicted in Fig. 1, in which segments of a unit circle are eliminated, and the remaining pieces form a new circle of smaller radius.

Another fractal example is (as often) of the Cantor set type (Mattila 1995, Section 4.10). Suppose from a unit circle the middle third segment $\left[\frac{2 \pi}{3}, \frac{4 \pi}{3}\right)$ is cut out, such that the two pieces $\left[0, \frac{2 \pi}{3}\right)$ and $\left[\frac{4 \pi}{3}, 2 \pi\right)$ remain, as is depicted in Fig. 2a. From these remaining pieces, the respective middle third segments are cut out again, as is depicted in Fig. 2b-e; and so on $a d$ infinitum. Thereby a continuum of measure zero is obtained: at the $n$ 'th construction stage, 
Fig. 1 An intuitive and informal example may help to understand why "cutting out holes" in a continuum might yield different radii if one "glues" together the remaining pieces. a Consider an original circle with radius 1 ; b pieces of $30^{\circ}$ are cut out of a, thereby effectively reducing the length of the set by a factor of two; c those pieces are "glued together" to yield a half-circle; $\mathbf{d}$ alternatively one can draw a full circle with a reduced radius of half the original radius

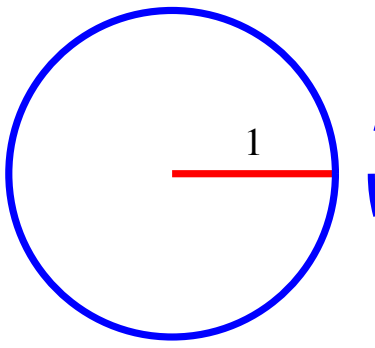

(a)

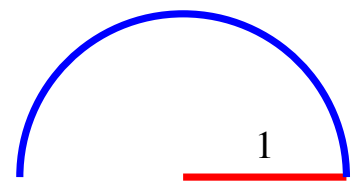

(d)

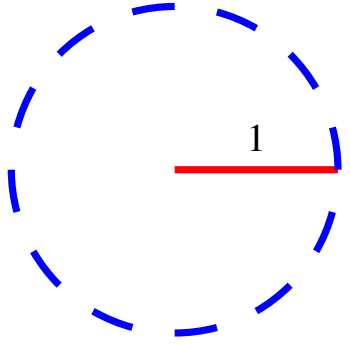

(b)

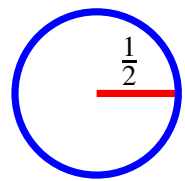

(c)

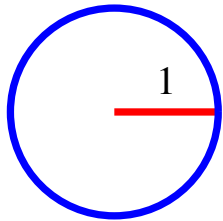

(a)

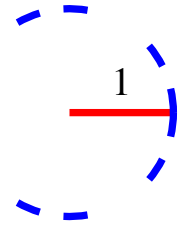

(d)

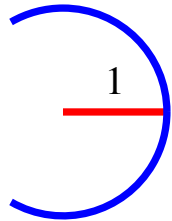

(b)

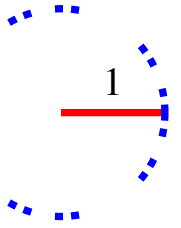

(e)

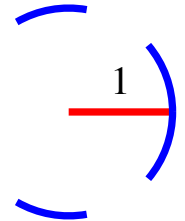

(c)

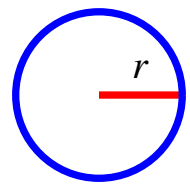

(f)

Fig. 2 A fractal example of why "cutting out holes" or "creating gaps" in a continuum in a scale invariant manner might yield different radii if the remaining pieces are scaled by the fractal dimension and subsequently "pasted" together. a Consider an original circle with radius 1 ; b the middle third segment $\left[\frac{2 \pi}{3}, \frac{4 \pi}{3}\right)$ is cut out of $\mathbf{a}$, thereby effectively reducing the length of the set by a factor of $\frac{1}{3}$; $\mathbf{c}$ the middle third segments $\left[\frac{2 \pi}{9}, \frac{4 \pi}{9}\right)$ and $\left[\frac{14 \pi}{9}, \frac{16 \pi}{9}\right)$ are cut out of the remaining segments in $\mathbf{b}$, thereby effectively reducing the length of the set by a factor of $\frac{1}{3} ; \mathbf{d}-\mathbf{e}$ shows the iteration of this construction; $\mathbf{f}$ alternatively one can draw a full circle with a pasting of the upscaled segments and a reduced radius $r \approx 0.8$ from Eq. (2)

encode each first remaining third by 0 and each third remaining third by 1 , and associate these respective bits with the $n$ 'th digits of a binary number. In the limit this construction creates the binary unit continuum $[0,1]$. However, at each construction stage, the set "loses" one third of its length, so that, in the limit this length converges to zero; that is, $\lim _{n \rightarrow \infty}\left(\frac{2}{3}\right)^{n}=0$. To avoid the scale dependence of the measure, Hausdorff introduced a non-integer exponential dimensional scale factor $d$ applied to the measure of the remaining pieces. This "dimension" $d$ 
is defined by an "Umklapp property" $d=\inf \left\{d \geq 0 \mid \lim _{n \rightarrow \infty}\left[2\left(\frac{1}{3}\right)^{d}\right]^{n}=0\right\}$, yielding $2^{n}\left(\frac{1}{3}\right)^{n d}=2^{n+1}\left(\frac{1}{3}\right)^{(n+1) d}$, and finally $d=\frac{\log (2)}{\log (3)}$.

So, effectively, the "price" of scale independence of the measure is the non-intuitive fact that the dimension of this set is not a natural number. In an ad hoc attempt to maintain some positive integer dimensionality of the set one may go one step further and attempt to change the metric. Thereby the intrinsic dimensional parameter is forced to become a natural number equal to or smaller than the dimension of the external embedding space.

For the sake of an example, note that the volume of a ball of radius $r$ in $d$-dimensional Euclidean space is $V(d, r)=(\sqrt{\pi} r)^{d} / \Gamma(d / 2+1)$. Suppose further that this measure of volume (which, strictly speaking, does not contain a dimensional parameter based upon Hausdorff's "Umklapp property" of the measure) nevertheless has an analytic continuation for real $d \geq 0$. Then, by "shifting" the dimensionality $d$ parameter to the "curvature" $r$; that is, by

$$
V(d, 1)=V(1, r)
$$

one obtains a "radius" $r$ associated with the Cantor set by inserting $d=\log 2 / \log 3$; that is,

$$
r=\frac{\pi^{\frac{d}{2}}}{2 \Gamma\left(\frac{d}{2}+1\right)}=\frac{\pi^{\frac{\log 2}{2 \log 3}}}{2 \Gamma\left(\frac{\log 2}{2 \log 3}+1\right)} \approx 0.8 .
$$

By abduction one may infer the following general desiderandum for the parametrization of "volume" as it relates to fractal dimensionality and curvature:

$$
V(d, R)=V(m, r) .
$$

Thereby the terms

1. fractal dimension $d$ on the left hand side of (3) refers to the dimension of the fractal object, as seen extrinsically, whereby the object is embedded in a space of extrinsic, higher dimensionality $n$;

2. outer, extrinsic curvature, parametrized by the radius $R$ on the left hand side of (3), stands for the curvature of the fractal object within an embedding space;

3. target dimension $m$ on the right hand side of (3), refers to the intrinsic dimension of the object "forced" to be a natural number; thereby the fractal set will, operationally and intrinsically, not be perceived as fractal but rather as a conventional continuum $\mathbb{R}^{m}$ of smaller or equal dimensionality than the embedding space, but of higher or equal dimensionality than the fractal; that is,

$$
d \leq m \leq n ;
$$

4. intrinsic curvature, parametrized by the radius $r$ on the right hand side of (3), refers to the curvature experienced intrinsically upon pretension of the target dimensionionality.

Corresponding to (4), as compared to the extrinsic radius, one obtains a smaller or equal intrinsic radius; that is

$$
R \geq r .
$$


Nottale (2011, Section 4.5) [for earlier discussions see Refs. Nottale (1993, Section 3.10) and 2001] and Nottale, Célérier, and Lehner have suggested a different, somewhat converse, "dual" approach by considering a scale relativity for gauge field theories, which is based upon (Nottale et al. 2006) "curvature at large scale and fractality at small scales." Thereby (Nottale 2011, Section 4.5.3, p. 129), "the metric elements and its curvature are everywhere explicitly scale dependent and divergent when the resolution scale tends to zero." This approach has been motivated by an a priori, given, fractal support of field theory. It presents no attempt to "re-encode" or "renormalize" the curvature and the metric in the presence of a fractal support such that this support intrinsically appear trivial in its topology.

Of course, these considerations are tentative, highly speculative and need further scrutiny. To quote a Referee, "the formal derivation remains an open question." Many issues and questions remain, among them how to conceptualize the shift (back \& forth) from the "fractality of the continuum" to the metric; and vice versa in more general situations. Also, it needs to be seen how to obtain curvature from an originally flat (zero curvature) spacetime. In the end, there might appear a possibility to extend the formalism of general relativity by "punching" scale invariant "holes" or "gaps" into space-time; thereby creating a theory of gravity which generalizes, or at least offers an alternative viewpoint to, relativity theory by assuming a fractal geometric support with non-curved standard metrics.

Acknowledgements Open access funding provided by TU Wien (TUW). The idea to fractal gravity emerged from a skype conversation of the author with Hui Deng, Tyler Hill and Barry Sanders on July 5th, 2017 on their beautiful paper (Hill et al. 2017) on light scattering in any dimension. Further formalizations were discussed and investigated with Ludwig Staiger and Cristian S. Calude. I kindly thank Karin Verelst for her interest in epistemological matters, and for her suggesting the quote by Henry Poincaré. I am grateful to Thomas Sommer for discussions and a critical reading of the manuscript. Nevertheless, I am to blame for all the misconceptions and omissions.

Open Access This article is distributed under the terms of the Creative Commons Attribution 4.0 International License (http://creativecommons.org/licenses/by/4.0/), which permits unrestricted use, distribution, and reproduction in any medium, provided you give appropriate credit to the original author(s) and the source, provide a link to the Creative Commons license, and indicate if changes were made.

\section{References}

Adda, F. B. (2007). Mathematical model for fractal manifold. International Journal of Pure and Applied Mathematics-IJPAM, 38, 155-186. arXiv:0711.3582.

Boskovich, R. (1966). De spacio et tempore, ut a nobis cognoscuntur. In J. M. Child (Ed.), A theory of natural philosophy (pp. 203-205). Cambridge, MA: MIT Press.

Bridgman, P. W. (1934). A physicist's second reaction to Mengenlehre. Scripta Mathematica, 2(101-117), 224-234.

Descartes, R. (1996). Meditations on first philosophy (J. Cottingham, Trans.). Cambridge: Cambridge University Press, p. 1641.

Edgar, G. (2008). Measure, topology, and fractal geometry. Undergraduate texts in mathematics (2nd ed.). New York: Springer.

Falconer, K. J. (2014). Fractal geometry: Mathematical foundations and applications (3rd ed.). Chichester: Wiley.

Hill, T., Sanders, B. C., \& Deng, H. (2017). Cooperative light scattering in any dimension. Physical Review A, 95, 033832 .

Hurewicz, W., \& Wallman, H. (1948). Dimension theory (Vol. 4)., Princeton mathematical series Princeton, NJ: Princeton University Press.

Jonas, S. (2016). Ineffability and its metaphysics. New York: Palgrave Macmillan US. 
Mandelbrot, B. B. (1982). The fractal geometry of nature. San Francisco: W.H. Freeman and Company.

Mattila, P. (1995). Geometry of sets and measuresin euclidean spaces: Fractals and rectifiability., Cambridge studies in advanced mathematics Cambridge: Cambridge University Press.

Montiel, E. M., Aguado, A. S., \& Zaluska, E. (1996). Topology in fractals. Chaos, Solitons \& Fractals, 7 , 1187-1207.

Nottale, L. (2011). Scale relativity and fractal space-time, Imperial College Press, distributed by World Scientific Publishing Co. Pte. Ltd. London, Singapore.

Nottale, L. (1993). Fractal space-time and microphysics: Towards a theory of scale relativity. Singapore: World Scientific.

Nottale, L. (2001). Scale relativity and gauge invariance. Chaos, Solitons \& Fractals, 12, 1577-1583.

Nottale, L., Célérier, M.-N., \& Lehner, T. (2006). Non-abelian gauge field theory in scale relativity. Journal of Mathematical Physics, 47, 032303. arXiv:hep-th/0605280.

Nottale, L., \& Schneider, J. (1984). Fractals and nonstandard analysis. Journal of Mathematical Physics, 25, 1296-1300.

Ord, G. N. (1983). Fractal space-time: A geometric analogue of relativistic quantum mechanics. Journal of Physics A: Mathematical and General, 16, 1869-1884.

Plato. (2000). In G. R. F. Ferrari (Ed.), The republic. Cambridge texts in the history of political thought (T. Griffith, Trans.). Cambridge: Cambridge University Press.

Poincaré, H. (1905). La valeur de la science. Paris: Flammarion (English translation: The value of science, 1907; German translation: Der Wert der Wissenschaft 1906).

Poincaré, H. (1913). The foundations of science-Science and hypothesis, value of science, science and method. Science and education series Vol. 1 (G. B. Halsted, Trans.). New York: The Science Press.

Porchon, H. (2012). Fractal topology foundations. Topology and Its Applications, 159, 3156-3170.

Putnam, H. (1981). Reason. Truth and history. Cambridge: Cambridge University Press.

Rogers, C. A. (1970). Hausdorff measures. Cambridge: Cambridge University Press.

Rössler, O. E. (1998). Endophysics. The world as an interface. Singapore: World Scientific.

Segal, A., \& Goldschmidt, T. (2018). The necessity of idealism. Idealism: New essays in metaphysics (pp. 34-49). Oxford: Oxford University Press.

Stace, W. T. (1934). The refutation of realism. Mind, 43, 145-155.

Svozil, K. (1986). Dimensional reduction via dimensional shadowing. Journal of Physics A: Mathematical and General, 19, L1125-L1127.

Svozil, K. (1987). Quantum field theory on fractal space-time. Journal of Physics A, 20, 3861-3875.

Svozil, K. (1994). Extrinsic-intrinsic concept and complementarity. In H. Atmanspacher \& G. J. Dalenoort (Eds.), Inside versus outside (Vol. 63, pp. 273-288)., Springer series in synergetics Berlin: Springer.

Svozil, K., \& Zeilinger, A. (1986). Dimension of space-time. International Journal of Modern Physics A, 1, 971-990.

Svozil, K., \& Zeilinger, A. (1988). Is there a breakdown of QED in ( $g-2)$-measurements? Physica Scripta, $T 21,122$

Toffoli, T. (1978). The role of the observer in uniform systems. In G. J. Klir (Ed.), Applied general systems research: Recent developments and trends (pp. 395-400). New York: Plenum Press.

Zeilinger, A., \& Svozil, K. (1985). Measuring the dimension of space-time. Physical Review Letters, 54, 2553-2555.

Publisher's Note Springer Nature remains neutral with regard to jurisdictional claims in published maps and institutional affiliations.

Karl Svozil is an Austrian physicist. He obtained is Ph.D. after studying in Vienna and Heidelberg. Currently he is Ao. Univ. Professor at the Institute for Theoretical Physics of the Vienna Technical University. He served as president of the International Quantum Structures Association (IQSA). His research is at the interface of the foundations of quantum theory and computation. He has published several papers in various areas of quantum theory including quantum non-locality and quantum randomness, and a number of books in quantum logic and related topics. 